\title{
Visions of a Wireless Future in Education Technology
}

\author{
Gavin Russell \& lan Pitt \\ University College Cork, Ireland
}

\section{g.russell@cs.ucc.ie i.pitt@cs.ucc.ie}

\begin{abstract}
This paper presents our research into the possibilities to wirelessly enhance classroom teaching in a university environment. We explore current technologies, both hardware and software, and propose a vision of future possibilities.
\end{abstract}

Keywords: Wireless, PDA, Education, Class Room Feed Back.

\section{Introduction}

Traditional lecture room teaching has numerous drawbacks. Students, for instance, don't always want to collaborate in the process. This may be because they are too shy, too tired or simply uninterested. Many teachers over the years have accepted the fact and teaching has sometimes become a one-way channel. Students arrive, take notes and leave. We often ask the age old questions, "Does everyone understand" or "Would anybody like me to go through this topic again" or our favorite dodge at the end of a lecture, "Any questions?" which is normally quickly followed by "Okay see you next week, good-bye" before the students have a chance to react.

This lack of feedback and response from students is contrary to one of the basic Seven Principles for Undergraduate Education created by Chickering and Gamson in 1987 (Chickering \& Stephen, 1996), that of encouraging communication between students and faculty.

This paper outlines possible teaching environments which would facilitate students in giving anonymous or known real-time feedback for the teacher. The teacher will see the feedback immediately and has a possibility to react depending on the comments given.

There are many possible solutions to this problem. We will first outline some of the existing solutions and the technologies involved, then propose our own system, and finally try to envisage what may arrive in the future.

Material published as part of this journal, either on-line or in print, is copyrighted by Informing Science. Permission to make digital or paper copy of part or all of these works for personal or classroom use is granted without fee provided that the copies are not made or distributed for profit or commercial advantage AND that copies 1) bear this notice in full and 2) give the full citation on the first page. It is permissible to abstract these works so long as credit is given. To copy in all other cases or to republish or to post on a server or to redistribute to lists requires specific permission from the publisher at Publisher@InformingScience.org 


\section{Existing Solutions}

\section{ClassTalk}

Since the early 1990s, many educators have been developing systems to improve classroom feedback to promote student learning. One such early system was ClassTalk developed by Better Education Inc.

Their motivation in developing the system was, "What can a teacher do in a class of 3 to 5 students that is difficult to do in a class of 30?". (Better Education, 1985). The answers they arrived at included:

- Interact with every student;

- Get all students actively involved;

- Understand what every student knows;

- Spot what each student doesn't know;

- Remedy problems in understanding when they occur;

- Check all homework;

- See what everyone is doing, and what they are not doing;

- Leave no student behind.

These observations were the basis of their initial system. The system was called the Classroom Communication System or ClassTalk and it is still available today, although no longer owned by Better Education Inc.

The system, while technical, is based on a very old but sound Socratic method of learning by answering questions, and works as follows. The students are presented with a question and a list of possible responses. After students choose an answer, the answers are collected and the sum of student responses is used to display a graph of overall response. Thus both the students and teacher can see the responses and, depending on the results, the teacher can decide whether it is necessary to discuss further how the answers were arrived at. If the response from the class is incorrect or wildly confused then the topic needs to be explained again, possibly in a different manner.

From a technical point of view, the system is based around scientific calculators with data ports hard-wired to a basic network. Further Information about the development of the system is contained in an excellent paper by Louis Abrahamson (Abrahamson, 1999).

\section{EduCue's PRS}

EduCue a Personal Response System was developed by Nelson Cue and C.K. Lee of HKUST (Hong Kong University of Science and Technology). This system uses a keypad and relies on the use of Infrared Technology to provide the feedback. All questions are posed in multiple-choice format and the user simply "beams" their answer at the receiver using an infrared keypad, much like changing channels on a Television. Student responses are collected, and the results displayed as a histogram using a data projector and a central screen.

This system has proved very successful and is used by many universities in the US and Asia. It has recently been enhanced to enable users to simplify the connection between one or more PRS infrared receivers and the computer. This is done using the wireless 802.11 standard which oper- 
ates in the $2.4 \mathrm{GHz}$ license free frequency range. Thus many receivers spread across a large area can all contribute to the overall results.

\section{More Modern Solutions}

While the previously-mentioned solutions do provide a basic feedback system, the emerging technologies of WLAN (Wireless Local Area Networks), Handheld computers (PDA's and Pocket PCs) and Cellular phones now present opportunities that developers in the early nineties could only have dreamed about. In his 1999 paper, Louis Abrahamson, wondered, “...Maybe by 2003, Java with wireless networking will be available on low-cost handhelds, that can be mass marketed to education". This day has now arrived and the possibilities are enormous.

Firstly we should perhaps look at what is happening in this emerging world of devices. We propose that there are five categories of new devices. We will quickly outline the five categories and then go into more depth with the final two, which we feel have the most potential.

1) PDA (personal digital assistants) with basic IR connectivity: These devices may be used in small groups and provide data exchange similar to the PRS system. These devices are difficult to network using IR and are best suited to small group tasks, or to allow students to work on solo tasks and then deliver their results to the teacher for assessment.

2) Cell Phone using WAP (Wireless application protocol): Most European Cell phones now come with WAP browsers which allow them to access WAP enabled websites. These websites are text based and would allow the teacher to implement a basic response system for students. We recently surveyed a first year humanities class taking an introductory CS module and found that of a class of 53 all but 1 owned a Cell phone with WAP capability. The main problem with using this system is the cost. While online the users are spending the same as they would on voice calls. This could amount to a cost of $€ 30$ for a one hour class. This is not a solution compatible with the majority of student's finances.

3) Cell Phones capable of downloading JAVA applications. We investigated the possibility of developing a JAVA application that would accumulate student response and send the result to a central server using the SMS (Small Messaging Service) available on the cellular network. This would cut the cost from $€ 30$ to approximately $€ 0.25$ but would remove the real-time feedback advantages of earlier systems. As an aside this system could be used to perform inclass tests with the students submitting their answers at the end.

4) Pocket PCs, such as the Hewlett Packard iPaq: These devices have significant advantages over the three previous devices.

a) A screen on which to display the questions; these screens are significantly bigger than those on cell phones.

b) Superior Processing Power, allowing the user to view animations or instructional videos.

c) Multiple networking options, such as WLAN and Bluetooth, which don't have the associated cost of cell phones running on telecom company networks.

5) The final group we looked at are at the bleeding edge of mobile devices. These new devices are based around the cell phones in group 3 but also have the addition of WLAN technology, thus removing the costs associated with using cellular networks. The potential of these devices is similar to that which we will outline below for the Pocket PC devices from category 4 (above) but there will be limitations on screen sizes. 


\section{Our Vision of the WLAN Enabled Pocket PC}

We first looked at a Hewlet Packard iPaq 5550. The advantages of this device can be seen in any pocket PC based PDA with wireless technology.

These devices provide connectivity via multiple channels including WLAN 802.11b(IEEE 2001), Bluetooth (Bluetooth, 2004), IrDA. This allows the devices to communicate with each other and with servers for campus communication without the costs associated with GSM Mobile Devices.

It also provides finger-print recognition, which would allow students to automatically login and have the device configured to their specific requirements. Thus everything could be stored on a server and devices would act like terminals. Students could just pick one up at the start of a lecture and return it at the end.

With suitable software, the devices could be used to support a variety of different types of interaction between student and lecturer, for example:

- Deliver 'Blackboard' style web-pages which give the student access to all material related to the course.

- Enable students to collaborate with one another using Bluetooth - for example sharing information, or working in groups, with each member tackling one part of a shared task or problem.

- Allow simple Yes/No Feed back to lecturer, who can then respond accordingly

- Allow students to indicate areas on photos delivered to the device (e.g., anatomical images in medical classes). Indications/selections can be transmitted to the lecturer for analysis/marking.

- Deliver Multiple Choice tests

- Deliver various types of class test/assessment, using the fingerprint recognition to verify student identity.

- Monitor attendance and participation - students would not be able to sign in for one another.

- A Group off-switch which can disconnect all users from the internet to prevent students surfing their way through a class.

- Using Bluetooth, students could walk up to a print station and obtain print outs of material held on the device - all charging could be done using identity verification

- Bluetooth could also be used to communicate with other devices, such as mobile phones, to allow the student to check email while off campus.

- The devices could also be used as a general-purpose student gadget/tool. Students could receive email without having to go to a lab, receive announcements (no more 'I didn't get it' excuses), etc.

- Deliver announcements - the sever can send information to device as soon as it is turned on, e.g., the device comes on, checks for new messages and, if device is on the server, sets some flag to notify the user that information is waiting to be collected (push technology)

- There is also the possibility of using voice over IP.

These are only a few of the possibilities. 
Providing such features on a hand-held device would also have a certain novelty, which might - at least initially - encourage student participation. Of course, this might also lead students to spend more time playing with the device rather than using it for its intended purpose. However, research suggests that such behaviour, while common, is usually short-lived (Chen, Myers \& Yaron, 2000).

The recent introduction of wireless internet to the public in Ireland could also contribute to the uptake of such systems. For example, a community centre could have a wireless or ADSL connection (with wireless router attached) to provide Internet Aware electronic classrooms in geographically remote locations. Head sets could be attached (via bluetooth) to provide audio feedback to remote lecturers.

In order to realise these possibilities, it is necessary to develop a system to take advantage of all this new technology.

One approach would be to develop a suite of applications to handle specific tasks, providing similar functionality to that currently provided by Blackboard or WebCT. However, the scope of these applications would need to be enormous if they are to support all the possibilities offered by wireless devices, and even then they would almost certainly constrain many users, forcing them to adapt their ideas to suit the tools available rather then utilising the medium in accordance with their own ideas.

What is needed is an architecture that provides a range of basic facilities and allows users to construct their own applications by combining and linking features as required. Such an architecture would provide:

- Automatic login and configuration of devices, including checks on ID.

- Transmission of content between wireless devices (individually or collectively) and the server and other central facilities (such as printers, scanners, etc.), using various wireless transmission mechanisms.

- Transmission of data between individual wireless devices or groups, using various wireless transmission mechanisms, with the possibility of monitoring by the lecturer.

- Access to Email

- A format that supports the various types of data to be handled by the system, e.g., text, images, sounds, movies, animations, etc.. This would need to be compatible with existing formats whilst providing additional functions to support wireless operation. An XMLderived language would be one option.

The format chosen would support scripting to allow the creation of content, e.g., for quizzes, simple animations, etc.. Examples and templates would be included to allow non-technical users to develop content for their courses. However, the use of scripting in an XML-based language would also allow more technically knowledgeable users to develop more sophisticated tests, animations, demonstrations, etc..

In choosing a format and designing the tools to support it, we have in mind the approach used in applications such as HyperCard. This would allow users to select a category that best represents their level of skill, e.g., 'authoring' 'scripting', etc. They could then interact with the material at the indicated level, e.g., changing the text in a pre-defined quiz to suit their subject, cutting-andpasting elements from examples to create interactive demos and animations, or scripting their own demos and animations from scratch. 


\section{Conclusion}

This paper has presented some of the history associated with using wireless devices as tools for improving the standard of education we deliver. We have also outlined our vision for the future possibilities or these devices. We are currently working on trial feedback systems and to carry out testing with our students over the coming months. Some of our students have already become aware of this research and are excited and already volunteering to get involved in the project.

\section{References}

Abrahamson, L. (1999). Teaching with classroom communication system - What it involves and why it works. 7th International Workshop "New Trends in Physics Teaching", Puebla, Mexico, May 27-30, 1999.

Better Education. (1985).Better Educations Vision. Retrieved December 1, 2003 from http://www.bedu.comindex.html

Bluetooth. (2004) An introduction to Bluetooth. Retrieved March 1, 2004 from https://www.bluetooth.org/bluetooth/landing/flash/intro.swf

Chickering, A. \& Stephen, C. E. (1996). Implementing the seven principles: Technology as lever. AAHE Bulletin, October 1996, pp. 3-6.

Chen, F., Myers, B., \& Yaron, D. (2000). Using handheld devices for tests in classes. Carnegie Mellon University School of Computer Science Technical Report, no. CMU-CS-00-152 and Human Computer Interaction Institute Technical Report CMU-HCII-00-101. July, 2000

IEEE Standard for Local and Metropolitan Area Networks: Overview and Architecture. Retrieved March 1, 2004 from http://standards.iee.org/getieee802/802.html

\section{Biographies}

Gavin Russell has been a lecturer in Computer Science, University College, Cork, Ireland since 1997. Before joining the university, he worked in the Web Publishing and Internet service provision businesses. His main research interests include: Web Based Learning, Interactive Multimedia on the World Wide Web and Secure Internet Payment Systems. He received an M.Sc. in Multimedia Technology in 2000 and is currently undertaking a Ph.D. in Computer Science at University College, Cork.

Ian Pitt is a Lecturer in the Department of Computer Science at U.C.C. He originally studied Music and Drama before going on to take a masters degree in Music Technology. He then took up a research post at the University of York, UK, working on the design of auditory humanmachine interfaces. After completing his D.Phil he spent a year as a post-doctoral research fellow at Otto-von-Guericke University, Magdeburg, Germany, before moving to Cork. His main research interests are speech, sound, and the design of human-machine interfaces, particularly multimedia interfaces and interfaces for use by blind people. 